\title{
ON NOETHERIANNESS OF NASH RINGS
}

\author{
FULVIO MORA AND MARIO RAIMONDO
}

\begin{abstract}
We introduce a class of rings, called Nash Rings, which generalize the notation of rings of Nash functions.

Let $k$ be any field, $X$ be a normal algebraic variety in $k^{n}$, and $U \subset X$. A Nash ring $D$ is the algebraic closure of $\Gamma\left(X, B_{X}\right)$ in a suitable domain $B$ such that $U$ is contained in the maximal spectrum of $B$ and $\Gamma\left(X, e_{X}\right)$ is analytically isomorphic to $B$ at each $x \in U$.

We show that $D$ is a ring of fractions of the integral closure of $\Gamma\left(X, C_{X}\right)$ in $B$. Moreover, if $k$ is algebraically nonclosed and if every algebraic subvariety $V \subset X$ intersects $U$ in a finite number of connected components (in the topology induced by $B$ ), then $D$ is noetherian.
\end{abstract}

Introduction. A Nash function on an open domain $U$ of $\mathbf{R}^{n}$ is a real analytic function defined on $U$ which is algebraic over $\mathbf{R}\left[X_{1}, \ldots, X_{n}\right]$, i.e. there exists $P \in \mathbf{R}\left[X_{1}, \ldots, X_{n}, T\right]$ such that $P(x, f(x))=0$ for all $x \in U$. The ring $N(U)$ of the Nash functions is the algebraic closure of $\mathbf{R}\left[X_{1}, \ldots, X_{n}\right]$ in the ring $O(U)$ of real analytic functions.

The ring $N(U)$ has been widely studied (cf. the references given in [1]), since it seems to give a useful tool in real algebraic geometry. In this paper we consider a class of rings (called Nash rings) which behave similarly to the ring of Nash functions on an open domain of $\mathbf{R}^{n}$. Let $k$ be any field, $X$ an irreducible, affine algebraic variety defined over $k$ (cf. [6]) and let $U$ be a subset of $X$; then a Nash ring (relative to $(X, U))$ is the algebraic closure $D$ of $\Gamma\left(X, \Theta_{X}\right)$ in a suitable domain $B$ (which will play the role of $O(U)$ ) (cf. §3) for more details).

The main results obtained in this work are the following:

(i) If $X$ is normal then $D$ is a ring of fractions of the integral closure of $\Gamma\left(X, \Theta_{X}\right)$ in $B$ (cf. Theorem 4.2).

(ii) If $X$ is normal and $k$ is algebraically nonclosed, then we give a sufficient condition in order to have $D$ noetherian (cf. Theorem 4.4).

These results extend, respectively, Corollary 3.1 of [1] and Theorem 2.1 of [5] in that we do not assume that $k=\mathbf{R}$ and that $X$ is nonsingular.

All of the above applies notably to the case when $X$ is a normal real variety, $U$ is an open (or compact) subset such that $B=\Gamma\left(U, A_{X}\right)$ is an integral domain (where $A$ denotes the sheaf of real analytic functions). So the Nash functions will be those analytic functions which verify an algebraic relation over the polynomials. We point out that in this paper there is no "semialgebraic type" assumption.

Received by the editors July 29, 1982 and, in revised form, May 25, 1983.

1980 Mathematics Subject Classification. Primary 14G30; Secondary 13E05.

Key words and phrases. Nash rings, noetherian property, algebraic closure of rings. 
1. We recall some definitions and properties of algebraic geometry on any field (for a general reference see [6]) and set some notations.

Let $k$ be any field, for a $k$-algebra $R$ we denote by $\Omega(R)$ the set of all maximal ideals of $R$ and by $\Omega_{k}(R)$ the subset of maximal ideals with residue field $k$. Both will be endowed with the topology induced by $\operatorname{Spec}(R)$. An affine algebraic variety is a pair $\left(X, \theta_{X}\right)$ where $X$ is a Zariski closed subset of some $k^{n}$ and $\theta_{X}$ is the sheaf of regular functions on $X$. We will denote $\Gamma_{X}=\Gamma\left(X, \theta_{X}\right)$ the ring of global sections of $\theta_{X}$ and we always consider the case where $X$ is irreducible, i.e. $\Gamma_{X}$ is an integral domain. It is well known that $\Gamma_{X} \simeq N^{-1} P$ where $P$ is an integral finitely generated $k$-algebras such that $\cap \mathrm{m}=(0)$ where $\mathrm{m} \in \Omega_{k}(P)$ and $N=\{f \in P \mid f \notin \mathrm{m} \forall m \in$ $\left.\Omega_{k}(P)\right\}$. It turns out that $\Gamma_{X}$ is an excellent ring (cf. [2, p. 259]). We will use the following important family of polynomials.

1.1. Proposition. Let $k$ be an algebraically nonclosed field. Then for each $n \in \mathbf{N}$ there exists a homogeneous polynomial $Q_{n} \in k\left[X_{1}, \ldots, X_{n}\right]$ such that $Q_{n}\left(x_{1}, \ldots, x_{n}\right)=$ 0 if and only if $\left(x_{1}, \ldots, x_{n}\right)=(0, \ldots, 0)$.

Proof. Cf. [6, Corollary 1, p. 31].

Finally, for any local ring $R$ we denote by ${ }^{h} R$ (resp. $R^{\wedge}$ ) the henselization (resp. the completion) of $R$ with respect to its maximal ideal. For a domain $A$ we will denote $A_{\circ}$ its quotient field and $\bar{A}$ the integral closure of $A$ in $A_{\circ}$.

2. In this section we deal with the local case. Let $(A, \mathfrak{m}),(B, \mathfrak{M})$ be local domains (not necessarily noetherian) such that $A \subset B$ is a local homomorphism and let $D$ be the algebraic closure of $A$ in $B$.

\subsection{Lemma. The ring $D$ is local with maximal ideal $\mathfrak{n}=\mathfrak{M} \cap D$.}

Proof. Let $a \in D, a \notin \mathfrak{m} \cap D$. Then $1 / a$ is an element of $B$ which is algebraic over $D$ and so $1 / a \in D$.

As in the following sections we will always suppose that the ring $A$ is excellent (cf. $\S 1$ ). We will also make this assumption for the next local results which actually hold under weaker algebraic assumptions, and restrict our study to the case of normal rings.

2.2. Proposition. Let $(C, \mathfrak{p})$ be a local domain such that $A \subset C \subset D$ with $\mathfrak{n} \cap C=\mathfrak{p}, \hat{A}=\hat{B}$ and $A$ a normal excellent domain. Then $C$ is noetherian (consequently $D$ is so).

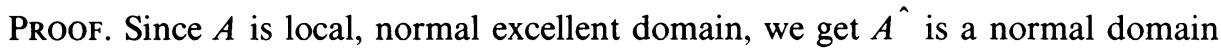
(cf. [2, Theorem 79, p. 258]). Thus by [3, 44.1, p. 188] the henselization ${ }^{h} A$ is algebraically closed in $A^{\wedge}$ and therefore it is the algebraic closure of $A$ in $\hat{A}$. We now have $\hat{C^{\wedge}} \simeq A^{\wedge}$ (since $\mathrm{m}=\mathfrak{p} \cap A$ ) so that ${ }^{h} C={ }^{h} A$ is a noetherian domain. Our contention follows then by the faithful flatness of $C \rightarrow{ }^{h} C$.

2.3. Proposition. Let $A$ be a normal excellent domain and let $E$ denote the integral closure of $A$ in $B$. Then $D \simeq S^{-1} E$ where $S=E-(\mathfrak{M} \cap E)$. 
Proof. Let $F$ be the integral closure of $A$ in $A^{\wedge}$ and $T=F-(\hat{\mathrm{m}} \cap F)$. We have ${ }^{h} A=T^{-1} F$ (cf. [4, Corollary 1, p. 127]); it is easy to check that the following diagram is a cartesian square.

$$
\begin{array}{ccc}
S^{-1} E & \rightarrow & D \\
\downarrow & & \downarrow \\
T^{-1} F & \rightarrow & { }^{h} A
\end{array}
$$

Then, both the lower and upper arrows are isomorphisms.

3. We now give the definition of a Nash ring as a generalization of the ring of Nash functions on an open domain of $\mathbf{R}^{n}$.

If $U$ is an open domain of $\mathbf{R}^{n}$, then the ring of Nash functions on $U, N(U)$ is the algebraic closure of $\mathbf{R}\left[X_{1}, \ldots, X_{n}\right]$ in the ring $O(U)$ of real analytic functions defined over $U$. It turns out that $N(U)$ is the algebraic closure of $\Gamma_{\mathbf{R}^{n}}$ in $O(U)$ and that for each $x \in U$ there exists a maximal ideal $\mathfrak{M}_{x} \in \Omega_{k}(O(U))$ such that $\left(\left(\Gamma_{\mathbf{R}^{n}}\right)_{\mathfrak{M},} \cap \Gamma_{\mathbf{R}^{n}}\right)^{\wedge}$ $\simeq\left(O(U)_{M,}\right)^{\wedge} \simeq \mathbf{R} \llbracket X_{1}, \ldots, X_{n} \rrbracket$. We use these properties as definition.

Let $k$ be any field, $X$ an irreducible affine algebraic variety defined over $k$ and let $A=\Gamma_{X}$. Let now $U \subset X$ be a subset. We say that a ring $D$ is a Nash ring relative to $(X, U)$ if

(1) there exists an integral domain $B$ with the properties

(i) $A \hookrightarrow B$ is a homomorphism of $k$-algebras,

(ii) there is an injection $U \hookrightarrow \Omega_{k}(B)$ such that if $x \mapsto M_{x}$ then

$$
\mathfrak{M}_{x} \cap A=\mathfrak{m}_{x}=\{f \in A \mid f(x)=0\},
$$

(iii) for each $x \in U$ we have $\left(A_{\mathrm{m}}\right)^{\wedge} \simeq\left(B_{M_{1}}\right)^{\wedge}$.

(2) $D$ is the algebraic closure of $A$ in $B$. Clearly, $D$ does not depend only on ( $X, U$ ) but also on the choice of $B$ which is not uniquely determined. We will also consider the following property for a ring $R$ with $A \subset R \subset B$ :

$(*)_{U} \quad f \in R$ is such that $f \notin \mathfrak{M}_{x} \cap R$ for all $x \in U$ then $1 / f \in R$.

3.1. Remarks. (i) If $B$ satisfies $(*)_{U}$ then $D$ does. In fact, if $f \in D, f \notin \mathfrak{M}_{x} \cap D$ for all $x \in U$ then $1 / f \in B$ and so if $1 / f \in D$ it is algebraic over it.

(ii) $U$ can be identified with a subset of $\Omega_{k}(D)$ as well as a subset of $\Omega_{k}(E)$, where $E$ is the integral closure of $A$ in $B$.

3.2. LeMMA. If the base field $k$ is algebraically nonclosed and $B$ satisfies $(*)_{U}$ then: $U \simeq \Omega_{k}(D) \simeq \Omega_{k}(B)$.

Proof. We prove that $\Omega_{k}(B) \simeq U$ and the same proof will work for $\Omega_{k}(D) \simeq U$. Let $\mathfrak{M} \in \Omega_{k}(B)$. Then $\mathfrak{M} \cap A=\mathfrak{m} \in \Omega(A)$ and $\mathfrak{m}=\left(g_{1}, \ldots, g_{t}\right) A$ (because $A$ is noetherian). Therefore there exists $x \in U$ such that $g_{i}(x)=0, i=1, \ldots, t$. Let $f \in \mathfrak{M}$ and $h=Q_{t+1}\left(f, g_{1}, \ldots, g_{t}\right)$ (cf. Proposition 1.1). Then $h \in \mathfrak{M}$. If for all $y \in U, h \notin \mathfrak{M}$, then $1 / h \in B$ and so $\mathfrak{M}=B$, a contradiction. Therefore there exists $y \in U$ such that $h \in \mathfrak{M}_{y}$; then $f(y)=0$ and $g_{i}(y)=0$ for $i=1, \ldots, t$. We conclude that $y=x$ and $\mathfrak{M}=\mathfrak{M}_{x}$. 
3.3. Proposition. Let $x \in U, \mathfrak{n}_{x}=\mathfrak{M}_{x} \cap D$ and $\mathfrak{m}_{x}=\mathfrak{M}_{x} \cap A$. Then $D_{11}$ is $a$ subring of the algebraic closure $G_{x}$ of $A_{\mathfrak{m}_{1}}$ in $B_{\mathfrak{M}_{1}}$. Moreover $D_{\mathrm{n}_{1}}$ and $G_{x}$ are noetherian local domains.

Proof. By Proposition 2.2 it is enough to prove that $D_{\mathrm{n}_{x}} \subset G_{x}$. Let $f=a / b \in D_{\text {u, }}$ with $a, b \in B \subset B_{\mathfrak{M}}$, and $b \notin \mathfrak{M}_{x}$, then $f \in B_{\mathfrak{M}}$. Since $b$ is algebraic over $A \subset A_{\text {m, }}$, we have $b \in G_{x}$ and also $1 / b \in G_{x}$ (since it is algebraic over it). Then $a / b=f \in G_{x}$.

4. From now on we suppose that $X$ is a normal variety (i.e. $A=\Gamma_{X}$ is a normal domain). We consider a Nash ring $D$ as given in $\S 3$. We also suppose that $B$ is normal and that $(*)_{U}$ holds.

4.1. Remark. The assumption that $B$ is normal ensures that $D$ is, as it is easily checked using the fact that $D \simeq D$ 。 $\cap$. The following theorem extends a result of [1, Corollary 3.1].

\subsection{Theorem. Let $S=\{f \in E \mid f(x) \neq 0 \forall x \in U\}$. Then $D=S^{-1} E$.}

Proof. Let $f \in D$ and let $K=(A[f])_{\circ}$; we denote by $A_{k}=D \cap K$ and by $G$ the integral closure of $A$ in $K$. Clearly, it is enough to prove that $A_{K}=T^{-1} G$ where $T=\{g \in G \mid g(x) \neq 0 \forall x \in U\}$. Since $D$ is normal (cf. Remark 4.1) we have $G \subset D$ and by $(*)_{U} T^{-1} G \subset D$, hence $T^{-1} G \subset A_{K}$.

Now let $A=\Gamma_{X} \simeq N^{-1} P$ be affine representation (cf. $\S 1$ ) and let $F$ be the integral closure of $P$ in $K$. Then $G=N^{-1} F$.

Let $H$ denote the integral closure of $P[f]$ in $K$. Then $F \subset H$, for a fixed $x \in U$; let $\mathfrak{a}_{x}=\{g \in H \mid g(x)=0\}$ and $\mathfrak{p}_{x}=\mathfrak{a}_{x} \cap F$. Since $F_{\mathfrak{p}_{x}}$ and $H_{\mathrm{a}_{x}}$ have isomorphic henselizations, we have (cf. [3, p. 187]) that $h t\left(a_{x}\right)=h t\left(p_{x}\right)$ and so $a_{x}$ is isolated in its fiber. By Zariski's Main Theorem (cf. [4, Theorem 1, p. 41]) it is easy to see that $F_{\mathfrak{p}_{x}} \simeq H_{\mathfrak{a}_{x}}$, hence $f \in F_{\mathfrak{p}_{x}}$. We conclude that $f \in \cap_{x \in U} F_{\mathfrak{p}_{x}}=M^{-1} F$ where $M=\{h \in$ $F \mid h(x) \neq 0 \forall x \in U\}$. Since $N \subset M$ we have $f \in T^{-1} G$.

In order to prove our main theorem we give the following version of the condition (P) given by Risler (cf. [5, p. 367]) in the context of rings of Nash functions.

(C) For all $\mathfrak{p} \in \operatorname{Spec}(A)$, the $\operatorname{ring} B / \mathfrak{p} B$ has a finite number of idempotents (i.e. $\operatorname{Spec}(B / p B)$ has a finite number of connected components).

\subsection{CorollaRy. If $k$ is algebraically nonclosed, then $\Omega(D) \simeq \Omega_{k}(D) \simeq U$.}

Proof. In fact, if $\mathfrak{n} \in \Omega(D), \mathfrak{n} \cap E=\mathfrak{n}^{\prime}$ is a maximal ideal of $E$ (since $D$ is a localization of $E$ ). By Going $\mathrm{Up}^{\prime} \cap A$ is a maximal ideal of $A$, then $\mathfrak{n} \cap A=\mathfrak{n}^{\prime} \cap$ $A=\mathrm{m}_{x}=\left(g_{1}, \ldots, g_{t}\right) A$ with $x \in X$. Now let $f \in \mathfrak{n}$ and let $h=Q_{t+1}\left(f, g_{1}, \ldots, g_{t}\right)$. Then there exists $y \in U$ such that $h(y)=0$ (if $h(y) \neq 0$ for all $y \in U$, then $h$ is a unit in $D$ ), therefore $g_{i}(y)=0$ for all $i=1, \ldots, t$ and so $y=x$ and $f \in \mathfrak{n}_{x}$. By Lemma 3.2 we have $U \simeq \Omega(D) \simeq \Omega_{k}(D) \simeq \Omega_{k}(B)$.

4.4. THEOREM. In the above setting if the base field $k$ is algebraically nonclosed and if condition (C) holds, then $D$ is noetherian. 
Proof. We first show that, for all $\mathfrak{p} \in \operatorname{Spec}(A)$ there is only a finite number of primes $\mathfrak{a}_{i}$ in $D$ lying over $\mathfrak{p}$ and such that $h t\left(\mathfrak{a}_{i}\right)=h t(\mathfrak{p})$. For all $\mathfrak{n} \in \Omega(D), D_{\mathfrak{n}}$ is noetherian by 3.3. Then for all $\mathfrak{q} \in \operatorname{Spec}(D), D_{\mathrm{a}}$ is a localization of a suitable $D_{11}$ and so it is noetherian.

Now let $\mathfrak{q} \in \operatorname{Spec}(D), \mathfrak{p}=\mathfrak{q} \cap A$ and $\mathfrak{n} \supset \mathfrak{q}$ be a maximal ideal of $D, h t(q)=$ $h t(\mathfrak{p})$ because $D_{\mathrm{n}}$ and $A_{\mathrm{n} \cap A}$ have the same henselization (cf. [3, p. 187]). Now $B \otimes_{A} k(\mathfrak{p})$ is a localization of $B \otimes_{A} A / \mathfrak{p}$ so that, by $(\mathrm{C}), B \otimes_{A} k(\mathfrak{p})$ has a finite number of idempotents; hence also $D \otimes_{A} k(p)$ has a finite number of idempotents. Now, for all $\mathfrak{q} \in \operatorname{Spec}(D)$ lying over $\mathfrak{p}$, since $D_{\mathfrak{q}}$ is noetherian and $h t(\mathfrak{q})=h t(\mathfrak{p})$, we have $\operatorname{dim}\left(D_{\mathfrak{q}} \otimes_{A_{\mathfrak{v}}} k(\mathfrak{p})\right)=0$ (cf. [2, Theorem 19]), then $D \otimes_{A} k(\mathfrak{p})$ is artinian (it is 0 -dimensional with a finite number of idempotents). We can conclude that there is a finite number of primes $\mathfrak{a}_{1}, \ldots, \mathfrak{a}_{s}$ lying over $\mathfrak{p}$. Moreover if $\mathfrak{p} D$ is primary (cf. [3, p. 187]) then $\mathfrak{p} D=\bigcap_{i=1}^{s} \mathfrak{a}_{i}$.

We now show that $n \in \Omega(D)$ are finitely generated, in fact $\mathrm{n}$ is the only maximal ideal of $D$ lying over $\mathrm{m}=\mathrm{n} \cap A=\left(g_{1}, \ldots, g_{t}\right) A$, hence $\mathrm{m} D=\left(g_{1}, \ldots, g_{t}\right) D$.

The conclusion of the noetherianness of $D$ follows as in Risler's proof (cf. [5, p. 372]), using descending induction on the height of prime ideals and the fact that if all primes of height $\geqslant h$ are finitely generated then all ideals of height $\geqslant h$ are so (cf. [5, Lemma 2.13]). Let $\mathfrak{q} \in \operatorname{Spec}(D)$ and $\mathfrak{p}=\mathfrak{q} \cap A$, then $\mathfrak{p} D=\mathfrak{q} \cap \mathfrak{q}_{1} \cap \cdots \cap \mathfrak{a}_{r}$ where $h t(\mathfrak{q})=h t(\mathfrak{p})=h t\left(\mathfrak{a}_{j}\right)$. The thesis follows using the exact sequence of $D$-modules.

$$
0 \rightarrow \mathfrak{p} D \rightarrow \mathfrak{q} \oplus\left(\mathfrak{q}_{1} \cap \cdots \cap \mathfrak{q}_{r}\right) \rightarrow \mathfrak{q}+\left(q_{1} \cap \cdots \cap \mathfrak{q}_{r}\right) \rightarrow 0 .
$$

We explicitly remark that we proved indeed a possibly stronger version of Theroem 4.4. More precisely we showed that

4.5. THEOREM. If $k$ is algebraically nonclosed then $D$ is noetherian if and only if $D / p D$ has a finite number of idempotents for every $p \in \operatorname{Spec}(D)$.

We do not know if the converse of Theorem 4.4 holds. For the case of rings of Nash functions are [5, Proposition 3.1].

\section{REFERENCES}

1. J. Bochnak and G. Efroymson, Real algebraic geometry and the 17th Hilbert problem, Math. Ann. 251 (1980), 213-241.

2. H. Matsumura, Commutative algebra, 2nd ed., Benjamin, New York, 1980.

3. M. Nagata, Local rings, Interscience, New York, 1962.

4. M. Raynaud, Anneaux locaux henséliens, Lecture Notes in Math., vol. 169, Springer-Verlag, Berlin and New York, 1970.

5. J. J. Risler, Sur l'anneau des fonctions de Nash globales, Ann. Sci. École Norm. Sup. (4) 8 (1975), 365-378.

6. A. Tognoli, Algebraic geometry and Nash functions, I.N.D.A.M. Inst. Math. 3 (1978).

Department of Mathematics, Istituto di Matematica dell'Universita di Genova, Via L. B. AlberTI 4-16132 GenOVA, ItALY 